\title{
Phenomenology 2.0
}

\section{Melvin Mclnnis (iD) | Holli Bertram (1D}

Department of Psychiatry, University of Michigan, Ann Arbor, Michigan

\section{Correspondence}

Melvin McInnis, 4250 Plymouth Road, Department of Psychiatry, University of Michigan, Ann Arbor, MI 48109.

Email:mmcinnis@umich.edu

An estimated 10,000 mental health apps are now available for download $^{1}$ to your mobile device. The appetite for digital and mobile technology related to health is undeniable. While a broad array of selection options in the marketplace is generally positive for consumers, many challenges exist in determining which of these apps are helpful. Most reach the market with limited information on utility and the majority have no research supporting the suggested uses or claims. The MONARCA group from Denmark, whose study is published in this issue, sets an excellent example of scientific rigor with their determination to test their technology and publish their findings. ${ }^{2}$

Mobile technology in health care has three primary uses. First, it acts as an information delivery system. Written information about human medical conditions, once limited to print materials, is now readily available electronically. Technology for transmitting and reading this information is in standard use.

The second use of mobile health technology is monitoring health states, exemplified by the MONARCA study. ${ }^{2}$ The information gathering systems are similar in content to clinical interactions, but in the personal e-world of the user with online central accessibility. Assessments are available in real time, as the moods or emotions are being lived.

The third use of mobile health technology is within the healthcare delivery system. There are insufficient numbers of providers to treat those in need and apps are being developed to provide many types of care, and include interactive therapies integrated with outcome monitoring. This type of technology fills a need, offering services that might otherwise not be available to individuals.

\section{CHALLENGES}

The challenges of electronic mobile health systems to provide information, monitor health, and deliver care reflect the challenges of health care in general. Information available to the public about health and treatment varies in quality and accuracy. An information glut exists and sifting through it all is an imposing task. We rely on professional (NAMI) or government (NIMH) agencies to provide standardized health information treatment guidelines that can be accessed electronically.
Ongoing data collection to evaluate health patterns will improve knowledge of health states and drive decisions on care. Yet apps which monitor symptoms can feel intrusive and lead to response fatigue. Reminders to complete a symptom checklist can quickly become irritating and then ignored. How do we motivate completion of assessment measures? Are the assessment measures valid and accurate measures of what is being monitored? What are the desired outcomes of using this technology? Can digital phenomenology ${ }^{3}$ provide objective measures of classic phenomenology ${ }^{4}$ ?

Care delivery apps empower individuals to self-care and broaden access to treatment but limited data exist on the efficiency and efficacy of the current offerings. In order for novel technology to take hold in healthcare, it must be rigorously evaluated and demonstrated to be effective. A commercial academic collaboration can be strength in this type of research. While several studies are currently underway, adaptation into mainstream mental health care awaits evidence of efficacy and improved outcomes. ${ }^{5}$

The MONARCA study has many landmark features and makes important strides in evaluating the uses of technology in mental health care. The study engages the participant with technology at a personal level in "real-world" care settings by asking current questions related to mood instability, a fundamental feature of bipolar disorder that is often difficult to capture by retrospective report. The focus on patient engagement and longitudinal outcomes are additional strong elements in this study. Interpreting results are challenging due to the relatively small numbers of participants and the heterogeneity of bipolar disorders. In addition, there is the tautological dilemma inherent in longitudinal outcomes studies: instability leads to/results from perceived stress, which leads to/results from diminished functioning, which leads to/results from instability. We are challenged to define cause and effect in outcomes research.

Going forward, the field will benefit from a combined approach that integrates entity and event data. Entity data result from interactive monitoring of clinical patterns that engage individual and care providers alike. The rigor and standards of past generations of research can be easily carried forward into the "e-world" of mobile technology with forethought and planning. Event data are gathered passively as events emerge and generate highly informative patterns 
of health and behavior. The capacity for monitoring personal, social, and vocational activities enhances our knowledge of the person, but determination of causality remains a challenge. Privacy risks are greater with this technology, particularly in a vulnerable population. How do we evaluate the "risk-benefit" balance in providing much needed care to humanity?

The emerging tools of the "e-world" would have pleased the likes of Kraepelin and Jaspers ${ }^{4}$ as they provide increased granularity in the study of phenomenology and provide the much-needed dimensionality that they recognized to be present.

\section{ORCID}

Melvin Mclnnis (iD https://orcid.org/0000-0002-0375-6247

Holli Bertram (iD https://orcid.org/0000-0001-9446-5121

\section{REFERENCES}

1. Torous J, Andersson G, Bertagnoli A, et al. Towards a consensus around standards for smartphone apps and digital mental health. World Psychiatry. 2019;18(1):97-98.

2. Faurholt-Jepsen M, Frost M, Busk J, et al. Is smartphone-based mood instability associated with stress, quality of life and functioning in bipolar disorder? Bipolar Disord. 2019. https://doi.org/10.1111/ bdi.12796 [Epub ahead of print]

3. Barrett PM, Steinhubl SR, Muse ED, Topol EJ. Digitising the mind. Lancet. 2017;389(10082):1877.

4. Jaspers K. General Psychopathology. Baltimore, MD: Johns Hopkins University Press; 1997.

5. Lipschitz J, Miller CJ, Hogan TP, et al. Adoption of mobile apps for depression and anxiety: cross-sectional survey study on patient interest and barriers to engagement. JMIR Ment Health. 2019;6(1):e11334. 